\title{
Associations between a posteriori defined dietary patterns and bone mineral density in adolescents
}

\author{
Teresa Monjardino $\cdot$ Raquel Lucas $\cdot$ \\ Elisabete Ramos • Carla Lopes • Rita Gaio • \\ Henrique Barros
}

Received: 3 October 2013/ Accepted: 24 April 2014/Published online: 8 May 2014

(c) Springer-Verlag Berlin Heidelberg 2014

\begin{abstract}
Purpose Dietary pattern analysis may uncover the joint effects of multiple dietary components on bone health, but such research is scarce and targets mostly adults.

Methods We quantified prospective associations between dietary patterns and bone mineral density (BMD) in 1,007 adolescents of a cohort born in 1990 and recruited at schools in Porto during the 2003/2004 school year. Forearm BMD was measured using dual-energy X-ray absorptiometry. Participants' dietary patterns were classified "Healthier", "Dairy products", "Fast food and sweets" and "Lower intake" according to previously identified patterns obtained in a larger sample of 1,489 participants using the K-means method. Using dietary patterns at 13 years old as the main exposure, associations were estimated cross-sectionally (with BMD at the age of 13) and prospectively (with annual BMD variation between 13 and 17 years), using linear regression coefficients adjusted for height, weight, energy intake and, in girls, for menarche age.
\end{abstract}

T. Monjardino $(\bowtie) \cdot$ R. Lucas $\cdot$ E. Ramos $\cdot$ C. Lopes

H. Barros

Department of Clinical Epidemiology, Predictive Medicine and Public Health, University of Porto Medical School, Porto,

Portugal

e-mail: teresam@med.up.pt

T. Monjardino - R. Lucas - E. Ramos - C. Lopes - R. Gaio ·

H. Barros

Institute of Public Health, University of Porto, Porto, Portugal

R. Gaio

Department of Mathematics, Science Faculty, Centre for Mathematics, University of Porto, Porto, Portugal
Results No significant associations between the a posteriori dietary patterns identified and mean BMD at 13 were found. However, among girls, adherence to a pattern characterized by low intake of energy and all food groups was negatively associated with annual BMD variation between 13 and 17 years [adjusted coefficient $(95 \% \mathrm{CI})$ $\left.-0.451(-0.827 ;-0.074) \mathrm{mg} \cdot \mathrm{cm}^{-2} \cdot \mathrm{year}^{-1}\right]$.

Conclusions Although results showed that, in girls, adherence to a "Lower intake" dietary pattern is associated with lower annual BMD variation throughout adolescence, overall, there were no consistent associations between dietary patterns and forearm BMD in adolescents.

Keywords Dietary patterns - Bone density .

Cohort study $\cdot$ Adolescence

\section{Introduction}

Low bone mineral density (BMD) is a major risk factor for fragility fracture [1,2]. The attained peak bone mass during early adulthood is an important determinant of BMD throughout life [3]. Therefore, maximizing that peak during adolescence may be an important strategy to decrease the risk of osteoporotic fractures later in life [4]. Beyond the importance of genetic determinants, environmental factors, such as diet, have been shown to modulate bone gain during childhood and adolescence [5]. Prospective research during adolescence may be particularly relevant because this life stage is a critical window in time for bone mineral accrual [6].

The most consistently followed approach to examine potential relationships between dietary intake and bone quality has focused on individual effect of nutrients, foods or food groups [7]. However, due to the conceptual and 
methodological limitations of such approach, namely confounding by the concomitant intake of other foods and nutrients, the examination of the joint effects of multiple dietary components has recently gained great interest $[8$, 9]. Dietary pattern analysis is an alternative approach to study the associations between diet and chronic diseases that aims to describe each person's diet according to combinations of foods consumed rather than attempting to disentangle the effects of specific nutrients or foods $[8,10,11]$.

Dietary patterns can be defined a priori, through the use of indices based on present scientific evidence and theoretical assumptions, or a posteriori, in which statistical methods, such as factor and cluster analysis, are used to generate patterns from dietary data collected from specific study populations [8]. Whereas several studies have assessed the association between a posteriori defined dietary patterns and indices of bone quality in adult populations [12-21], little was investigated in children and adolescents [7, 22-26]. Since it takes into account overall food choices as they occur in the population without making any assumption of protective or harmful health effects of diet, a posteriori dietary pattern analysis may provide new and more realistic insights into the role of diet in bone health during this period when the greatest BMD accrual occurs.

Using data from a population-based cohort, our objective was to quantify the prospective associations between a posteriori dietary patterns in early adolescence and forearm BMD.

\section{Materials and methods}

Data were collected as part of the Epidemiological Health Investigation of Teenagers in Porto (EPITeen populationbased cohort). This project was designed to study growth, development and health in a population-based cohort of urban adolescents recruited in the schools of Porto, Portugal, and followed from early adolescence up to adulthood [27].

Cohort recruitment (13 years of age)

In the 2003/2004 school year, the executive boards of every school in Porto providing teaching to adolescents born in 1990 (27 public and 24 private) were contacted. All public schools and 19 private schools (79\%) agreed to participate. Executive boards were asked to provide contact information for each student's family. We identified 2,787 eligible adolescents, and 2,160 (1,651 public and 509 private schools students) agreed to participate and contributed at least for part of the planned assessment. Participation at the individual level was $78 \%$, similar in public and private schools (77.7 vs. $76.7 \%$, respectively, $P=0.60$ ). Legal guardians and adolescents received written and oral information explaining the purpose and the design of the study, and written informed consent was obtained from both. The study was approved by the Ethics Committee of the University Hospital of São João, Porto, and policies and procedures were developed to guarantee data confidentiality and protection. Sampling procedures and detailed methods have been described elsewhere [28].

Follow-up evaluation (17 years of age)

The first follow-up evaluation of the cohort took place in the 2007/2008 school year. Re-evaluations were scheduled by contacting schools or participants directly. Written informed consent was again obtained from all participants and their guardians. We re-evaluated 1,716 participants $(79.4 \%)$.

Data collection procedures

Baseline and follow-up evaluations were conducted using the same protocol. Data were collected by a team of trained health professionals through the use of two self-administered structured questionnaires (one completed at home, another at school) and a physical examination performed at school.

\section{Physical examination}

Forearm BMD $\left(\mathrm{g} \cdot \mathrm{cm}^{-2}\right)$ was measured at the distal radius of the non-dominant forearm by DXA using a Lunar $^{\circledR}$ Peripheral Instantaneous X-ray Image (PIXI) device (GE Medical Systems, Madison, WI, USA). In case of reported previous fracture of the non-dominant arm, the dominant arm was the one assessed. Short-term in vivo precision was calculated according to the recommendations of the International Society for Clinical Densitometry using two measurements for each 30 consecutive adolescents, and root mean square SD was $0.022 \mathrm{~g} \cdot \mathrm{cm}^{-2}$ at a $95 \%$ confidence level [29].

Anthropometrics were performed with subjects in light clothing and barefoot under standard procedures. Body weight was measured to the nearest tenth of kilogram (Tanita Corporation, Tokyo, Japan), and height was measured in centimetres, to the nearest tenth, using a portable stadiometer (Seca Deutschland, Hamburg, Germany). BMI was calculated as weight in kilograms divided by the squared height in metres.

Since examination at school limited conditions to evaluate adolescents' sexual maturity according to Tanner stages, self-reported age at menarche was used as a sexual development indicator for girls. 


\section{Questionnaire variables}

Adolescents were asked to indicate the frequency of spending at least 20 consecutive minutes in sports activities, beyond compulsory school activities [30], and we classified four levels of physical activity practice: never; $\leq 1$ times per week; 2-3 times per week; $>3$ times per week.

Parental education was measured as the number of successfully completed years of formal schooling, and adolescents were classified according to the parent with the highest educational level.

Dietary intake was evaluated at 13 years of age, in the baseline evaluation, using a FFQ designed according to Willett and colleagues and validated in the Portuguese adult population. The FFQ was then adapted for adolescents by including foods more frequently eaten by this age group [31]. The FFQ comprises 91 food or food group items and an open-ended section to add foods not listed in the questionnaire and eaten at least once per week. Respondents were asked to report how often they usually consumed each particular food over the prior 12-month period as the number of times per day, week or month. Each subject had to report the average frequency of consumption for each food item by choosing among nine categories, ranging from "never or less than once a month" to "six or more times a day". Seasonal variation in food consumption was considered by multiplying the frequency and portion of seasonal items by a seasonal factor of 0.25 (i.e. equivalent to consumption during a 3-month period). Since the adolescents FFQ version did not include specific questions on portion size, a standard portion size was considered for nutrient calculation. The frequency reported was multiplied by the previously defined average portion size to estimate the intake in grams or millilitres. Food consumption was converted into total energy intake with the software Food Processor Plus (ESHA Research, Salem, OR, USA 1997) based on values from the USDA and updated with values for typical Portuguese foods computed using the Portuguese Tables of Food Composition, typical recipes and data from previous studies [32].

\section{Dietary patterns definition}

Dietary patterns were previously identified in 1,456 participants with data on FFQ [33]. Briefly, the individual food items and the foods listed in the open-ended section of the FFQ were aggregated into 14 food groups according to nutritional similarities providing dairy products, fish, red meat, white meat, vegetables, added fats, fruits, pasta/ potatoes/rice, soft drinks, cereals, soup, coffee/tea, fast food and sweets.

Patterns were identified through the K-means method enhanced by bootstrapping (repeated random sampling with a fixed number of times) and the choice of different centres. This method categorizes individuals into mutually exclusive groups based on differences/similarities in dietary intake. For each sampling, the K-means algorithm with a number of clusters ranging from 1 to 6 was applied to 10 sets of different centres obtained randomly. The final model was the one presenting the lowest sum of squares of the intra-cluster distances (to the centres) [34]. The analysis was not stratified by gender since no significant effect of that variable on the intakes was identified.

Based on the 14 food groups, standardized caloric contribution of each food group was used as input variable. Here, the caloric contributions corresponded to the effect of each food group on caloric intake, multiplied by the food group intake.

Four dietary patterns, significantly different regarding mean food group intakes, were identified and named "Healthier", "Dairy products", "Fast food and sweets" and "Lower intake".

The "Healthier" pattern was characterized by the highest consumption of fish, vegetables and added fats. This pattern also presented high consumption of fruits and pasta/potatoes/rice and the lowest consumption of fast food and soft drinks. The "Dairy products" pattern was characterized by the highest consumption of dairy products and an intermediate consumption of most food groups. The pattern characterized by the highest energy intake and by the highest intake of sweets, fast food, soft drinks and coffee/tea was named "Fast food and sweets". Lastly, a pattern with low intake of all food groups, particularly of red meat, fish, fruits, pasta/potatoes/rice, dairy products, cereals and added fats was named "Lower intake". The characteristics of each pattern were similar in both genders but girls in the "Lower intake" pattern had also significantly lower intake of white meat and soup.

\section{Data analysis}

Categorical and continuous variables were summarized by percentages and mean $\pm \mathrm{SD}$, respectively. Differences in proportions were tested with the chi-squared test. One-way ANOVA and the Kruskal-Wallis test were used to compare continuous variables between independent samples. ANOVA was used to compare food groups, micronutrients and energy-adjusted protein intakes across dietary clusters. Bonferroni correction was used for multiple pair-wise comparisons. Using the identified dietary patterns as the main exposure and forearm BMD at 13 years of age $\left[\mathrm{BMD}_{13}\left(\mathrm{mg} \cdot \mathrm{cm}^{-2}\right)\right.$ and the annual BMD variation between 13 and 17 (annual BMD variation $\left.\left(\mathrm{mg} \cdot \mathrm{cm}^{-2} \cdot \mathrm{year}^{-1}\right)\right]$ as the outcomes, associations were estimated using linear regression coefficients and $95 \% \mathrm{CI}$. The "Healthier" pattern was used as reference. BMD was used in $\mathrm{mg} \cdot \mathrm{cm}^{-2}$ to improve 
readability. In addition to crude estimates, linear regression coefficients were adjusted for the major confounders: BMI, total energy intake, parental education and adolescents' regular practice of physical activity. In girls, coefficients were further adjusted to menarche age to address confounding by sexual maturity (third model). Data were analysed using Stata ${ }^{\circledR} 9.0$.

From 1,716 adolescents who agreed to participate in both evaluations of the EPITeen study, 333 participants were excluded because they did not have a valid measurement of forearm BMD and 347 participants had missing or invalid FFQ data. An additional 29 participants were excluded because they were missing information on one or more of the anthropometric variables or on menarche status.

A total of 1,007 (543 girls and 464 boys) had complete information on forearm BMD, anthropometric variables, diet and, in girls, on age at menarche (also excluding outliers for the definition of dietary patterns).

Comparing participants with complete information with those excluded from these analyses, we found no differences in sex distribution, BMD and BMI values. Adolescents with complete information had higher median (25th75th \%o) parental education [11 (8-16) vs. 9 (6-12) years; $P<0.001]$.

\section{Results}

In Table 1 , non-dietary characteristics at 13 years of age are compared between adolescents with different dietary patterns. Adolescents with "Healthier" and "Dairy products" dietary patterns were more likely to have parents with higher education. In girls, the "Lower intake" dietary pattern had the highest BMI, while in boys there were no significant differences in mean BMI according to dietary pattern. The frequency of physical activity practice did not significantly differ across dietary patterns.

In Table 2, consumption of food groups and nutrients that have traditionally been associated with bone density is described according to dietary patterns. Adolescents with a "Healthier" pattern had significantly higher consumption of vegetables and vitamin C. Besides having higher consumption of dairy products, adolescents in the "Dairy products" pattern had higher intake of vitamin $\mathrm{K}$, although not statistically significant. The "Fast food and sweets" pattern presented a significantly higher consumption of energy, soft drinks and phosphorus and, in boys, of vitamin D. The "Lower intake" pattern was characterized by significantly lower consumption of energy, fruits and, particularly in boys, of dairy products. Adolescents with this pattern had also the lowest intakes of calcium, phosphorus and vitamins $\mathrm{C}, \mathrm{D}$ and $\mathrm{K}$.
Table 3 summarizes the cross-sectional and prospective associations between BMD and dietary patterns by gender presenting crude and adjusted linear regression coefficients and $95 \%$ CI. Both crude and adjusted linear regression coefficients show no association between the dietary patterns identified and BMD at 13 years. However, in girls, a statistically significant inverse association was found between the "Lower intake" pattern and annual BMD variation between 13 and 17 years old [adjusted coefficient $(95 \%$ CI $\left.)-0.451(-0.827 ;-0.074) \mathrm{mg} \cdot \mathrm{cm}^{-2} \cdot \mathrm{year}^{-1}\right]$. Further adjustment for parental education and adolescents' regular practice of physical activity did not affect the results.

\section{Discussion}

To the best of our knowledge, this is the first study to explore cross-sectional and prospective associations between a posteriori defined dietary patterns and BMD during adolescence, the most critical period for bone mass development. We found no significant effect of the dietary patterns identified in this sample of adolescents on forearm BMD at 13 years of age. Regarding longitudinal associations, the dietary patterns identified in early adolescence were not associated with BMD at 17 years old (data not shown), but we observed that girls with a "Lower intake" dietary pattern had lower forearm annual BMD variation throughout adolescence.

The negative association between the "Lower intake" pattern and BMD annual increase observed in girls might be due to the combined effect of generalized low consumption and decreased vegetables, fruits and dairy products intake in this dietary pattern. Our findings are in line with previous longitudinal studies in adult women that have found significant positive associations between patterns with high intake of fruits, vegetables and/or dairy products and gains in bone physical properties [25, 35].

Among adults, a few studies dealt with the relationship between bone quality indices and dietary patterns, generated by data reduction techniques [12-21], but in children published work is still limited [7, 22-26]. We identified five studies in children and adolescents aiming to identify dietary patterns related to bone mass. In 2006, Li et al. [22] identified, by factor analysis, four dietary patterns in a sample of Korean children. A higher score in a "shellfish and processed meat" pattern was significantly positively associated with BMD in boys but not in girls. Additionally, the "pizza and drinks" dietary factor score was significantly positively associated with BMD only in girls. In 2009 , the association between dietary patterns at 6 and 12 months, defined by principal component analysis, and bone size and density at 4 years of age was quantified in a 


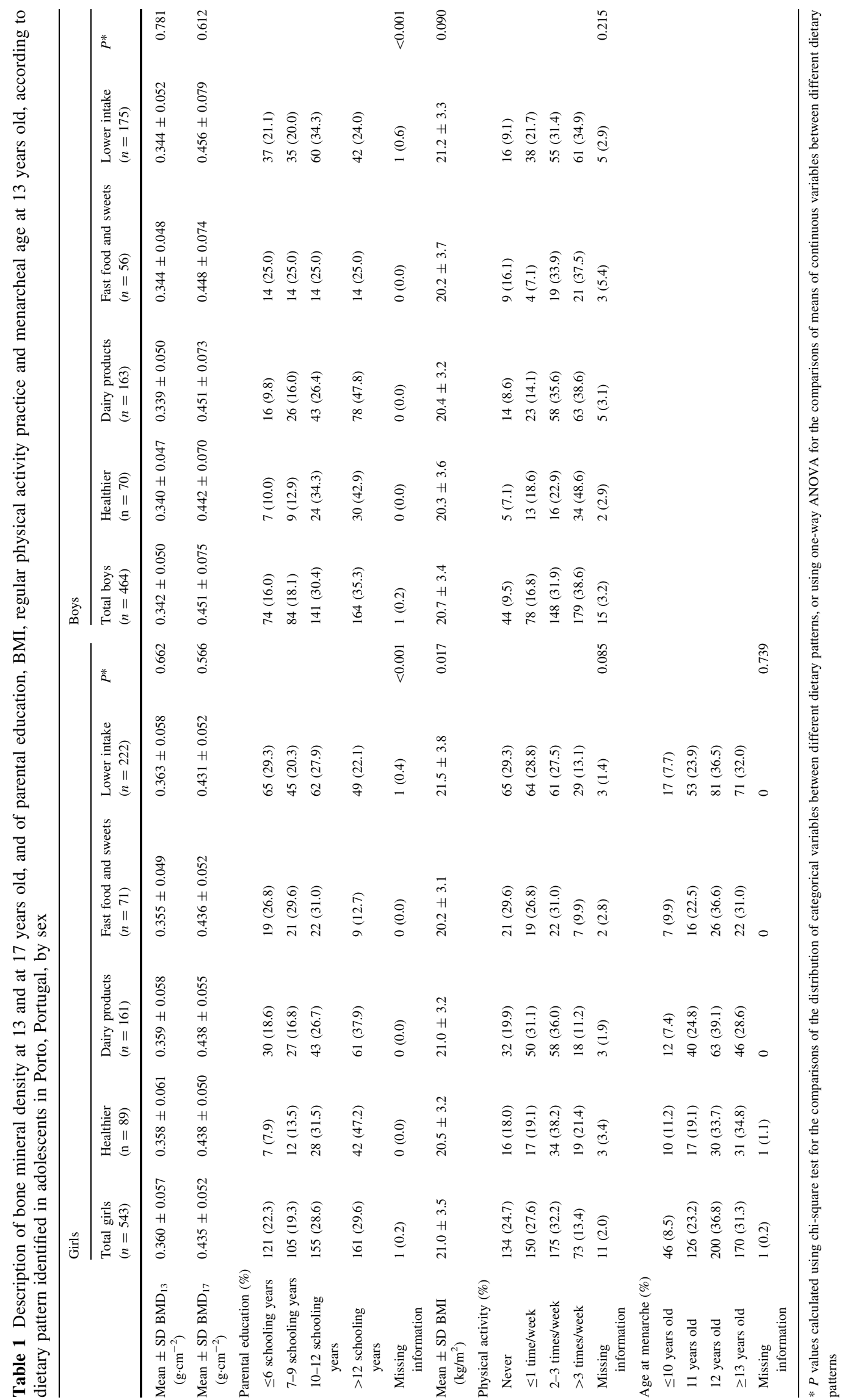




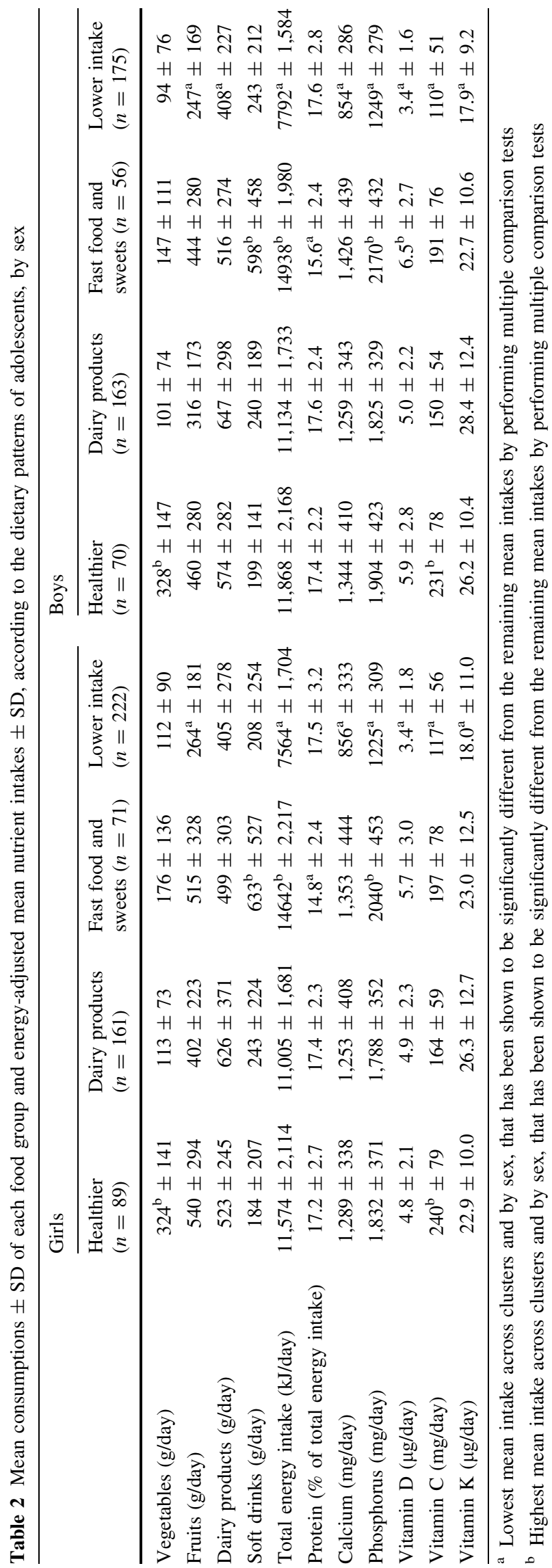

sample from the UK [23]. The degree of compliance with a pattern characterized by high consumption of vegetables, fruits and home-prepared foods was neither associated with bone area nor with bone mineral content (BMC) or BMD. In another study in the USA among children during the age period of 3.8-7.8 years, two dietary patterns were identified by reduced rank regression analyses, and a pattern characterized by increasing intake of vegetables and limited fried food intake was associated with higher BMD [24]. Noh et al. [25] identified dietary patterns by reduced rank regression associated with BMC in a sample of Korean elementary school girls. Higher scores in a pattern characterized by high consumption of fruits, nuts and seeds, and dairy products had more increased BMC over 22 months. Recently, Shin et al. [26] identified four dietary patterns by factor analysis in a sample of 196 Korean adolescents aged 12-15 years. Adolescents in the highest tertile of the "milk and cereal" dietary pattern had significantly lower likelihood of having low BMD compared with those in the lowest tertile.

In our study, we cannot rule out the possibility that the significant negative association between "Lower intake" pattern and BMD accrual, found in girls, was related to their higher BMD at 13 years of age. Since these girls had also higher BMI at baseline, the load effect on bone until the age of 13 may have led to earlier skeletal maturation, constraining further BMD increase [36]. Also, the trend towards weight loss throughout adolescence in these heavier girls (data not shown), and consequent reduction in mechanical exposure, may have determined lower BMD accrual. In addition, since these girls with a "Lower intake" pattern practiced less regular physical activity at 13 years of age, and a age-related decline trend in exercise is expected, low physical activity levels may have also conditioned smaller BMD increase [37]. We also hypothesized that "Lower intake" pattern girls are more likely to develop eating disorders and/or may be biologically older at 13 years of age, since they had higher BMD, and could have less room for BMD improvement between 13 and 17 years of age. However, the proportion of girls underweight [38] and mean age at menarche was not significantly different among girls over different dietary patterns (data not shown).

The small gender differences found in the association between the "Lower intake" pattern and annual BMD variation can be attributable to distinct biological maturity stages in girls and boys at the same chronological age, in which it is likely that biological determinants act differently on BMD. These differences may also be attributable to a sex-specific skeletal response to environmental determinants during adolescence, as previously described for the relationship between physical activity and BMD [39]. 
Although a significant negative association between the pattern with lower consumption of vegetables, fruits and dairy products and annual BMD variation in girls has been observed, individual effects of these food groups on mean BMD were not observable (data not shown). This is consistent with the hypothesis of the existence of cumulative and interactive effects between foods, disclosing the importance of dietary patterns analysis to study diet and health associations.

Dietary patterns can be defined a priori or a posteriori [8]. Since the definition of a posteriori patterns is not based on the assumption of protective or deleterious health effects of food components, this approach acknowledges that clustering of food components is culturally determined and can vary across populations and reflect the current population's food consumption [34, 40, 41]. Additionally, from a public health perspective, this approach provides information for setting nutritional interventions targeted to specific groups within a community that have similar patterns of diet [42]. However, specificities of each setting, namely the variety of dietary assessment methods and of statistical techniques used, make comparisons across existing studies difficult. Nevertheless, heterogeneity between studies regarding patterns associated with increased or decreased risk remains noteworthy.

It should be noted that the empirical identification of dietary patterns by explanatory approaches reflects dietary behaviour of the population rather than known or expected health effects of diet. Also, if populations have no dietary patterns characterized by higher intake of nutrients/foods that potentially influence bone properties, the effect of these individual diet components would be diluted by all patterns. Consequently, the identified dietary patterns are not necessarily relevant for bone health [43], which may be an important justification for the absence of associations, as happened in the present study [44].

Furthermore, the lack of association between diet and BMD at 13 years of age may reflect smaller effects of dietary determinants on $\mathrm{BMD}$ in comparison with genetic and early life determinants [3, 45]. It is possible that diet may not have the same impact on BMD in adolescents as it does in adult populations. Additionally, compared to previous studies of dietary patterns and bone health, the homogeneity of our sample of adolescents, born in the same year and living in the same city, may have hampered the finding of significant associations between diet and BMD [46].

The exclusion of individuals with lower parental education, because of missing data, contributed to homogeneity among participants. Nevertheless, since we observed no association between parental education and BMD (data not shown) and participants with lower parental education are more likely to have a "Lower intake pattern", we 
hypothesized that non-exclusion of these participants could have improved the statistical power for detecting a stronger association between this pattern and BMD.

We cannot also exclude the possibility that inadequate sample size may have led to an insufficient power of our study to detect significant associations.

Limitations of the dietary assessment method, affecting both precision and accuracy of the exposure measurement, may have also precluded identification of consistent associations. Invalid reporting of usual intake is a common shortfall $[47,48]$, which can limit dietary patterns analyses [47] and consequent detection of diet-disease relationships [49]. Moreover, a prespecified portion size in the FFQ may yield biased estimates of absolute energy and nutrients intake. Despite all that, the majority of variation in food intake is captured by frequency of consumption [50] and FFQ has been argued as a valid method in dietary pattern analysis in terms of the reproducibility of the patterns produced [51, 52]. Although the lack of validation of the FFQ in this adolescent population may have biased the assessment of dietary intake, we believe that this potential bias had a very low effect because the FFQ had been previously validated for adults of the same city [32].

In this study, we do not know whether dietary intake, evaluated at baseline, changed throughout the follow-up. While some studies report variation in dietary patterns during adolescence [53, 54], it is also likely that dietary patterns remain relatively constant overtime [55-58].

We used forearm BMD measured by DXA to estimate bone strength. Although we acknowledge that DXA measures partially reflect bone size rather than its volumetric density [59], BMD accurately predicts fracture risk during puberty [60]. However, forearm BMD represents a peripheral component of bone density, and we cannot rule out possible associations of other measures of bone quality, namely whole-body or at the axial skeleton, with the dietary patterns identified.

Dietary patterns are highly determined by cultural influences, and food choices are part of a larger aggregation of health behaviours. Indeed, dietary patterns seem to represent an overall lifestyle, in which the relative importance of each determinant is clearly difficult to measure in isolation [47]. Despite the fact that we were unable to detect important confounding in our sample, we cannot rule out that other lifestyle factors may have biased our results, either towards or away from the null.

The present dietary patterns were identified in urban Portuguese adolescents, and the results found may not be fully generalizable to other populations. However, by prospectively evaluating a large representative and homogeneous sample of adolescent from a high-income country, we have provided new insight regarding the role of dietary patterns in this critical period for bone health optimization, unaffected by cohort or period effects. We have identified a low overall intake diet as a possible negative influence on the achievement of the full genetic potential for skeletal mass during adolescence.

\section{Conclusions}

Although in girls, a "Lower intake" dietary pattern was negatively associated with bone annual variation during adolescence, overall, no consistent associations between dietary patterns identified through cluster analysis and forearm BMD in 13-year-old adolescents were found.

Acknowledgments The authors gratefully acknowledge Associação Portuguesa de Osteoporose (APO) for making the bone densitometry equipment available for both evaluations.

Conflict of interest The authors have no conflict of interest to disclose.

\section{References}

1. Woolf AD, Pfleger B (2003) Burden of major musculoskeletal conditions. Bull World Health Organ 81:646-656

2. Marshall D, Johnell O, Wedel H (1996) Meta-analysis of how well measures of bone mineral density predict occurrence of osteoporotic fractures. BMJ 312(7041):1254-1259

3. Heaney RP, Abrams S, Dawson-Hughes B, Looker A, Marcus R, Matkovic V, Weaver C (2000) Peak bone mass. Osteoporos Int 11(12):985-1009

4. Johnston CC, Slemenda CW (1994) Peak bone mass, bone loss and risk of fracture. Osteoporos Int 4(Suppl 1):43-45

5. McGuigan FE, Murray L, Gallagher A, Davey-Smith G, Neville CE, Van't Hof R, Boreham C, Ralston SH (2002) Genetic and environmental determinants of peak bone mass in young men and women. J Bone Miner Res 17(7):1273-1279. doi:10.1359/jbmr. 2002.17.7.1273

6. Weaver CM (2008) The role of nutrition on optimizing peak bone mass. Asia Pac J Clin Nutr 17(Suppl 1):135-137

7. Kontogianni MD, Yiannakouris N (2009) Diet and bone healththe perspective of dietary pattern analysis. Eur Musculoskelet Rev 4(1):73-74

8. Hu FB (2002) Dietary pattern analysis: a new direction in nutritional epidemiology. Curr Opin Lipidol 13(1):3-9

9. Kant AK (2004) Dietary patterns and health outcomes. J Am Diet Assoc 104(4):615-635. doi:10.1016/j.jada.2004.01.010

10. Tucker KL (2010) Dietary patterns, approaches, and multicultural perspective. Appl Physiol Nutr Metab 35(2):211-218. doi:10. 1139/H10-010

11. Jacques PF, Tucker KL (2001) Are dietary patterns useful for understanding the role of diet in chronic disease? Am J Clin Nutr 73(1):1-2

12. Tucker KL, Chen H, Hannan MT, Cupples LA, Wilson PW, Felson D, Kiel DP (2002) Bone mineral density and dietary patterns in older adults: the Framingham Osteoporosis Study. Am J Clin Nutr 76(1):245-252

13. Okubo H, Sasaki S, Horiguchi H, Oguma E, Miyamoto K, Hosoi Y, Kim MK, Kayama F (2006) Dietary patterns associated with bone mineral density in premenopausal Japanese farmwomen. Am J Clin Nutr 83(5):1185-1192 
14. Kontogianni MD, Melistas L, Yannakoulia M, Malagaris I, Panagiotakos DB, Yiannakouris N (2009) Association between dietary patterns and indices of bone mass in a sample of Mediterranean women. Nutrition 25(2):165-171. doi:10.1016/j.nut. 2008.07.019

15. Hardcastle AC, Aucott L, Fraser WD, Reid DM, Macdonald HM (2010) Dietary patterns, bone resorption and bone mineral density in early post-menopausal Scottish women. Eur J Clin Nutr 65(3):378-385. doi:10.1038/ejen.2010.264

16. Langsetmo L, Poliquin S, Hanley DA, Prior JC, Barr S, Anastassiades T, Towheed T, Goltzman D, Kreiger N (2010) Dietary patterns in Canadian men and women ages 25 and older: relationship to demographics, body mass index, and bone mineral density. BMC Musculoskelet Disord 11:20. doi:10.1186/14712474-11-20

17. McNaughton SA, Wattanapenpaiboon N, Wark JD, Nowson CA (2011) An energy-dense, nutrient-poor dietary pattern is inversely associated with bone health in women. J Nutr 141(8):1516-1523. doi:10.3945/jn.111.138271

18. Fairweather-Tait SJ, Skinner J, Guile GR, Cassidy A, Spector TD, MacGregor AJ (2011) Diet and bone mineral density study in postmenopausal women from the TwinsUK registry shows a negative association with a traditional English dietary pattern and a positive association with wine. Am J Clin Nutr 94(5):1371-1375. doi:10.3945/ajcn.111.019992

19. Whittle CR, Woodside JV, Cardwell CR, McCourt HJ, Young IS, Murray LJ, Boreham CA, Gallagher AM, Neville CE, McKinley MC (2012) Dietary patterns and bone mineral status in young adults: the Northern Ireland Young Hearts Project. Br J Nutr 1-11. doi:10.1017/S0007114511006787

20. Pedone C, Napoli N, Pozzilli P, Rossi FF, Lauretani F, Bandinelli S, Ferrucci L, Antonelli-Incalzi R (2011) Dietary pattern and bone density changes in elderly women: a longitudinal study. J Am Coll Nutr 30(2):149-154

21. Karamati M, Jessri M, Shariati-Bafghi SE, Rashidkhani B (2012) Dietary patterns in relation to bone mineral density among menopausal Iranian women. Calcif Tissue Int 91(1):40-49. doi:10.1007/s00223-012-9608-3

22. Li SJ, Paik HY, Joung H (2006) Dietary patterns are associated with sexual maturation in Korean children. $\mathrm{Br} \mathrm{J}$ Nutr 95(4):817-823

23. Harvey NC, Robinson SM, Crozier SR, Marriott LD, Gale CR, Cole ZA, Inskip HM, Godfrey KM, Cooper C (2009) Breastfeeding and adherence to infant feeding guidelines do not influence bone mass at age 4 years. Br J Nutr 102(6):915-920. doi:10. 1017/S0007114509317420

24. Wosje KS, Khoury PR, Claytor RP, Copeland KA, Hornung RW, Daniels SR, Kalkwarf HJ (2010) Dietary patterns associated with fat and bone mass in young children. Am J Clin Nutr 92(2):294-303. doi:10.3945/ajcn.2009.28925

25. Noh HY, Song YJ, Lee JE, Joung H, Park MK, Li SJ, Paik HY (2011) Dietary patterns are associated with physical growth among school girls aged 9-11 years. Nutr Res Pract 5(6):569-577. doi:10.4162/nrp.2011.5.6.569

26. Shin S, Hong K, Kang SW, Joung H (2013) A milk and cereal dietary pattern is associated with a reduced likelihood of having a low bone mineral density of the lumbar spine in Korean adolescents. Nutr Res 33(1):59-66. doi:10.1016/j.nutres.2012.11.003

27. Ramos E (2006) Health determinants in porto adolescents. PhD thesis. University of Porto, Porto

28. Ramos E, Barros H (2007) Family and school determinants of overweight in 13-year-old Portuguese adolescents. Acta Paediatr 96(2):281-286

29. Lucas R, Ramos E, Severo M, Barros H (2011) Potential for a direct weight-independent association between adiposity and forearm bone mineral density during adolescence. Am J Epidemiol 174(6):691-700. doi:10.1093/aje/kwr131

30. Aires L, Silva G, Martins C, Santos MP, Ribeiro JC, Mota J (2012) Influence of activity patterns in fitness during youth. Int J Sports Med 33(4):325-329. doi:10.1055/s-0031-1297955

31. Araujo J, Severo M, Lopes C, Ramos E (2011) Food sources of nutrients among 13-year-old Portuguese adolescents. Public Health Nutr 14(11):1970-1978. doi:10.1017/S1368980011 001224

32. Lopes C, Aro A, Azevedo A, Ramos E, Barros H (2007) Intake and adipose tissue composition of fatty acids and risk of myocardial infarction in a male Portuguese community sample. J Am Diet Assoc 107(2):276-286. doi:10.1016/j.jada.2006.11.008

33. Teixeira J (2011) Dietary patterns identification in Porto adolescents. Master thesis, University of Porto, Porto (in Portuguese)

34. Newby PK, Tucker KL (2004) Empirically derived eating patterns using factor or cluster analysis: a review. Nutr Rev 62(5): 177-203

35. Nieves JW, Melsop K, Curtis M, Kelsey JL, Bachrach LK, Greendale G, Sowers MF, Sainani KL (2010) Nutritional factors that influence change in bone density and stress fracture risk among young female cross-country runners. PM R 2(8):740-750. doi:10.1016/j.pmrj.2010.04.020

36. Judex S, Donahue LR, Rubin C (2002) Genetic predisposition to low bone mass is paralleled by an enhanced sensitivity to signals anabolic to the skeleton. FASEB J 16(10):1280-1282. doi:10. 1096/fj.01-0913fje

37. Brodersen NH, Steptoe A, Boniface DR, Wardle J (2007) Trends in physical activity and sedentary behaviour in adolescence: ethnic and socioeconomic differences. $\mathrm{Br} \mathrm{J}$ Sports Med 41(3):140-144. doi:10.1136/bjsm.2006.031138

38. Kuczmarski RJ, Ogden CL, Guo SS, Grummer-Strawn LM, Flegal KM, Mei Z, Wei R, Curtin LR, Roche AF, Johnson CL (2002) 2000 CDC growth charts for the United States: methods and development. Vital Health Stat Ser 11 Data Natl Health Surv 246:1-190

39. Macdonald H, Kontulainen S, Petit M, Janssen P, McKay H (2006) Bone strength and its determinants in pre- and earlypubertal boys and girls. Bone 39(3):598-608. doi:10.1016/j.bone. 2006.02.057

40. Oliveira A, Rodriguez-Artalejo F, Gaio R, Santos AC, Ramos E, Lopes C (2011) Major habitual dietary patterns are associated with acute myocardial infarction and cardiovascular risk markers in a southern European population. $\mathrm{J}$ Am Diet Assoc 111(2):241-250. doi:10.1016/j.jada.2010.10.042

41. Waijers PM, Feskens EJ, Ocke MC (2007) A critical review of predefined diet quality scores. Br J Nutr 97(2):219-231. doi:10. 1017/S0007114507250421

42. Pryer JA, Nichols R, Elliott P, Thakrar B, Brunner E, Marmot M (2001) Dietary patterns among a national random sample of British adults. J Epidemiol Community Health 55(1):29-37

43. van Dam RM (2005) New approaches to the study of dietary patterns. Br J Nutr 93(5):573-574

44. Michels KB, Schulze MB (2005) Can dietary patterns help us detect diet-disease associations? Nutr Res Rev 18(2):241-248. doi:10.1079/NRR2005107

45. Javaid MK, Cooper C (2002) Prenatal and childhood influences on osteoporosis. Best Pract Res Clin Endocrinol Metab 16(2):349-367. doi:10.1053/beem.2002.0199

46. Michels KB (2003) Nutritional epidemiology_past, present, future. Int J Epidemiol 32(4):486-488

47. Togo P, Osler M, Sorensen TI, Heitmann BL (2001) Food intake patterns and body mass index in observational studies. Int J Obes Relat Metab Disord 25(12):1741-1751. doi:10.1038/sj.ijo. 0801819 
48. Livingstone MB, Black AE (2003) Markers of the validity of reported energy intake. J Nutr 133(Suppl 3):895S-920S

49. Freedman LS, Schatzkin A, Midthune D, Kipnis V (2011) Dealing with dietary measurement error in nutritional cohort studies. J Natl Cancer Inst 103(14):1086-1092. doi:10.1093/jnci/ djr189

50. Noethlings U, Hoffmann K, Bergmann MM, Boeing H (2003) Portion size adds limited information on variance in food intake of participants in the EPIC-Potsdam study. J Nutr 133(2):510-515

51. Hu FB, Rimm E, Smith-Warner SA, Feskanich D, Stampfer MJ, Ascherio A, Sampson L, Willett WC (1999) Reproducibility and validity of dietary patterns assessed with a food-frequency questionnaire. Am J Clin Nutr 69(2):243-249

52. Khani BR, Ye W, Terry P, Wolk A (2004) Reproducibility and validity of major dietary patterns among Swedish women assessed with a food-frequency questionnaire. J Nutr 134(6):1541-1545

53. Madruga SW, Araujo CL, Bertoldi AD, Neutzling MB (2012) Tracking of dietary patterns from childhood to adolescence. Rev Saude Publica 46(2):376-386

54. Patterson E, Warnberg J, Kearney J, Sjostrom M (2009) The tracking of dietary intakes of children and adolescents in Sweden over six years: the European Youth Heart Study. Int J Behav Nutr Phys Act 6:91. doi:10.1186/1479-5868-6-91
55. Cutler GJ, Flood A, Hannan P, Neumark-Sztainer D (2009) Major patterns of dietary intake in adolescents and their stability over time. J Nutr 139(2):323-328. doi:10.3945/jn.108.090928

56. te Velde SJ, Twisk JW, Brug J (2007) Tracking of fruit and vegetable consumption from adolescence into adulthood and its longitudinal association with overweight. $\mathrm{Br} \quad \mathrm{J}$ Nutr 98(2):431-438. doi:10.1017/S0007114507721451

57. Mikkila V, Rasanen L, Raitakari OT, Pietinen P, Viikari J (2005) Consistent dietary patterns identified from childhood to adulthood: the cardiovascular risk in Young Finns study. Br J Nutr 93(6):923-931

58. Oellingrath IM, Svendsen MV, Brantsaeter AL (2011) Tracking of eating patterns and overweight - a follow-up study of Norwegian schoolchildren from middle childhood to early adolescence. Nutr J 10:106. doi:10.1186/1475-2891-10-106

59. Crabtree N, Ward K (2009) Bone densitometry: current status and future perspectives. Endocr Dev 16:58-72. doi:10.1159/ 000223689

60. Flynn J, Foley S, Jones G (2007) Can BMD assessed by DXA at age 8 predict fracture risk in boys and girls during puberty? An eight-year prospective study. J Bone Miner Res 22(9):1463-1467. doi:10.1359/jbmr.070509 